\title{
FRAM for CTA
}

\author{
P. Janeček ${ }^{\mathrm{a}}$, M. Prouza, and J. Ebr \\ Institute of Physics of the Academy of Sciences of the Czech Republic, Na Slovance 1999/2, 18221 Praha 8, \\ Czech Republic
}

\begin{abstract}
The Cherenkov Telescope Array (CTA) is a project to build a new generation ground-based gamma-ray observatory. Among other goals, the project aims to achieve high precision of gamma-ray energy measurement while maximizing the use of observation time. These objectives require detailed and fast information about atmospheric conditions, particularly the transparency which includes cloudiness (including thin high-altitude clouds) and aerosol content. This knowledge is required not only to select and calibrate data after observation, but also to make on-the-fly scheduling decisions. To provide such data without interfering with the observation (as would be the case when using laser-based methods), we propose to use the "FRAM" $(\mathrm{F}(/ \mathrm{Ph})$ otometric Robotic Atmospheric Monitor) device, which is a small robotic astronomical telescope with a large field of view and a sensitive CCD camera. FRAM will use stellar photometry to measure atmospheric extinction across the field of view of the CTA. The fast robotic mount of the telescope allows quick observation of multiple fields when the array is split and even a check of the conditions in the directions of the upcoming observations. The FRAM concept is built upon experience gained with a similar device operated at the Pierre Auger Observatory.
\end{abstract}

\section{Introduction}

When finished, the Cherenkov Telescope Array (CTA) will be by far the largest ground-based IACT (Imaging Atmospheric Cherenkov Telescope) gamma-ray observatory ever built [1], with two sites (one in each hemisphere) equipped by about a hundred telescopes of different sizes in total. To provide the groundbreaking astrophysical insights expected from such an enormous project, the CTA will rely not only on its sheer size, but also on high quality of the acquired data - to this end, strict requirements have been set that mandate a certain level of precision for each physical parameter. The maximal allowed error for Cherenkov light intensity has been set at $8 \%$ with an aim to lower the error to as little as $5 \%$ if possible. As there are several sources of uncertainties alongside the atmospheric conditions (such as the calibration of various parts of the detector), the error budget for each individual contribution is extremely thin and thus the uncertainty of the Cherenkov light intensity due to aerosol content of the atmosphere must be lower than 2\%. Moreover, as the CTA also aims for maximally efficient use of its observation time, scheduling decisions will be taken on-the-fly, requiring accurate knowledge of immediate atmospheric conditions in various directions across the sky.

To meet both these goals, a barrage of atmospheric monitoring is planned to be installed at both CTA sites, such as automated weather stations, all-sky cameras, ceilometers, the UVscope and LIDARs. From these devices, the LIDAR provides the most accurate information about both cloud and aerosol distribution in a given direction, with good resolution in height. However, the invasive nature of LIDAR operation (which amounts to shooting a laser to the sky) limits their use in the vicinity of the very sensitive IACTs if a reasonable amount of

a e-mail: janecekp@fzu.cz interference with observations is to be kept. On the other hand, the all-sky cameras, provide, by means of stellar photometry, a non-invasive measurement of clouds and aerosols in all directions, but with limited spatial resolution and sensitivity.

\section{The FRAM concept}

To bridge the gap between these two kinds of instruments, we propose to install on both CTA sites a device (which we call FRAM - F(/Ph)otometric Robotic Atmospheric Monitor) which consists of an astronomical CCD camera attached to a suitable photographic lens on an equatorial mount. The measurement principle - using apparent brightness of stars to infer atmospheric extinction or presence of clouds - of FRAM is the same as in the case of the all-sky cameras, but tailoring the angular size of the field of view (by the choice of the lens) to the parameters and configuration of the CTA telescopes allows for maximization of sensitivity and spatial resolution, while the use of a fast robotic mount means that FRAM can still always cover the direction of the CTA observation (or alternate between more directions, in case of split-array observations).

A big advantage of the non-invasive FRAM measurement is that it can be performed with high temporal resolution and within the actual field of view of the IACTs. However, everything comes with a cost - as the stars are located outside the atmosphere, only the integral extinction is measured with no information on the altitude profile. While FRAM will be able to detect the thinnest of clouds or aerosol layers, it cannot provide any insight on their height. Thus, in essence, FRAM is able to certify clear conditions and call upon other instruments in case of hazy ones.

At minimum, one FRAM would be installed at each CTA site. However, particularly for the southern site,

This is an Open Access article distributed under the terms of the Creative Commons Attribution License 4.0, which permits unrestricted use, distribution, and reproduction in any medium, provided the original work is properly cited. 


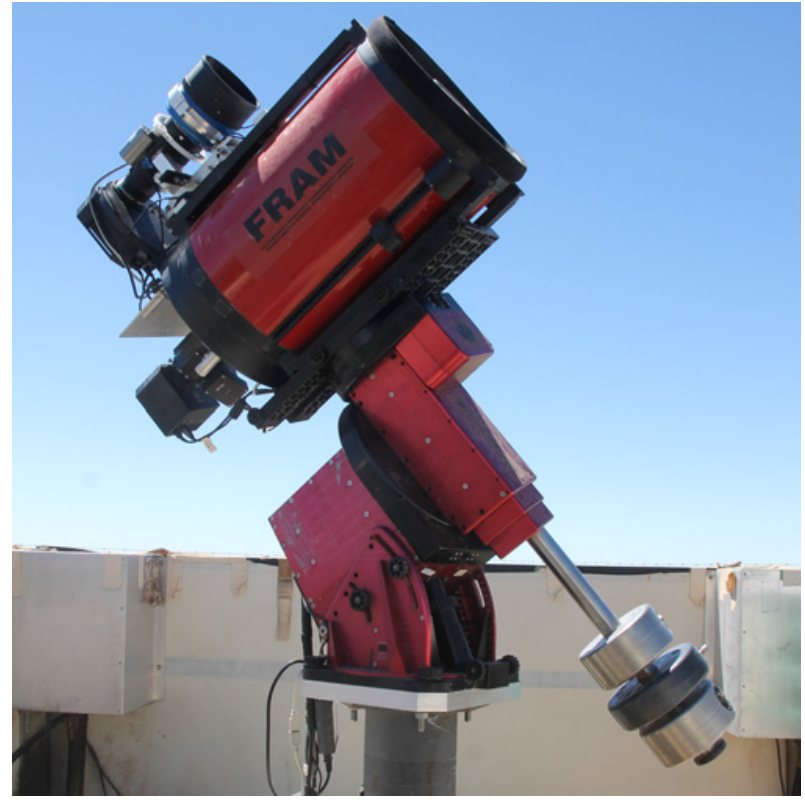

Figure 1. FRAM at the Pierre Auger Observatory.

which will span $10 \mathrm{~km}^{2}$ (as opposed to the $1 \mathrm{~km}^{2}$ of the northern site), installation of more FRAMs could be beneficial in order to minimize the distance between to the nearest FRAM for as many CTA telescopes as possible, as this distance introduces possibly an uncertainty in the measurement in case of horizontaly non-uniform atmosphere or scattered clouds. As an added bonus, with more FRAM devices, we can attempt to perform stereoscopic reconstruction of the clouds' positions in order to estimate their height (but it should be noted that the feasibility of this has not been tested in practice yet).

\section{FRAM at the Pierre Auger Observatory}

The design of the concept is based upon the FRAM robotic telescope [2] that the project group has built at the Pierre Auger Observatory in Argentina [3] in 2005 and operated in the harsh remote conditions of the Argentinean pampa ever since (Fig. 1). The Pierre Auger Observatory is the largest astroparticle observatory in the world and it includes 27 telescopes to observe the fluorescence light generated during the passage through the atmosphere of extensive air showers initiated by ultra-high energy cosmic rays. The goals of the Auger FRAM are very similar to that of the FRAM device we are proposing for the CTA. Even the ability of rapidly following a changing target area has been thoroughly tested on Auger in the so-called Shootthe-Shower program [4].

However there are conceptual differences between the two setups as the Fluorescence telescopes of Auger have an extremely wide field of view, covering a significant portion of the sky: concurrent monitoring of such an area would require essentially an all-sky camera. The Auger FRAM uses two ways to combat this issue: the first is to sample atmospheric properties with high precision at different directions using well-characterized stellar fields and extrapolate these data assuming spatial and temporal uniformity of the atmospheric conditions, the second is to do detailed follow-up (Shoot-the-Shower) observations covering wider, but still limited areas of the sky immediately after detection of particularly interesting air showers. The measurements of the first kind are carried out using a narrow-field camera mounted on a $30-\mathrm{cm}$ astronomical telescope (which is also used for other observations, such as GRB follow-ups), while those of the second kind use a wide-field camera connected to a photographic lens. The envisioned CTA FRAM retains only the wide-field camera, as the smaller CTA field can be reasonably covered at once, allowing the use of a lighter mount and in general reducing the complexity of operation and maintenance of the device.

\section{Hardware and operation}

Based on the extensive experience obtained at the Auger FRAM (where we have during the years tested several hardware models), we consider building the CTA FRAM mainly from the same or similar (commercially available) components as the latest configuration of the Auger FRAM [5].

- Paramount-MX robotic astronomical mount, a slightly down-scaled version of the Paramount-ME mount used by the Auger FRAM, which allows for completely remote operation and can smoothly resume observations even after a power cut thanks to absolute sensors that allow it to re-gain orientation autonomously.

- Moravian Instruments G4-16000 large-format CCD camera (with $36 \times 36-\mathrm{mm}$ sensor) equipped with standard BVRI photometric filters.

- Zeiss 135 f/2.0 photographic lens. This particular combination of sensor size and lens focal length provides a 15 degree field of view and thus covers the whole expected CTA field of view (up to 7 degrees) with a sufficient buffer to account for parallax between the FRAM and the more distant telescopes; the Zeiss lens is essentially unrivaled in aperture and sharpness at this focal length, particularly considering the large sensor area to cover.

- Rigel nStep focuser which mechanically couples to the manual focus wheel of the lens and thus allows remote re-focusing.

- Housing with a roof that can be opened/closed remotely, a control PC, control electronics for the roof and other auxiliary devices. Here again most of the design from the Auger FRAM can be used, but a switch from a custom-build hydraulic system to a commercial astronomical enclosure is being considered, mainly for budgetary reasons, as the custom-built hydraulic system of the Auger FRAM has proven to be extremely reliable.

The whole setup is constructed with autonomous operation without any need for human intervention in mind - again, the Auger experience is valuable here, as the Auger FRAM is located at a remote location, separated by a 30 minute drive from the nearest technician (availability of whom is not guaranteed on a short notice). 


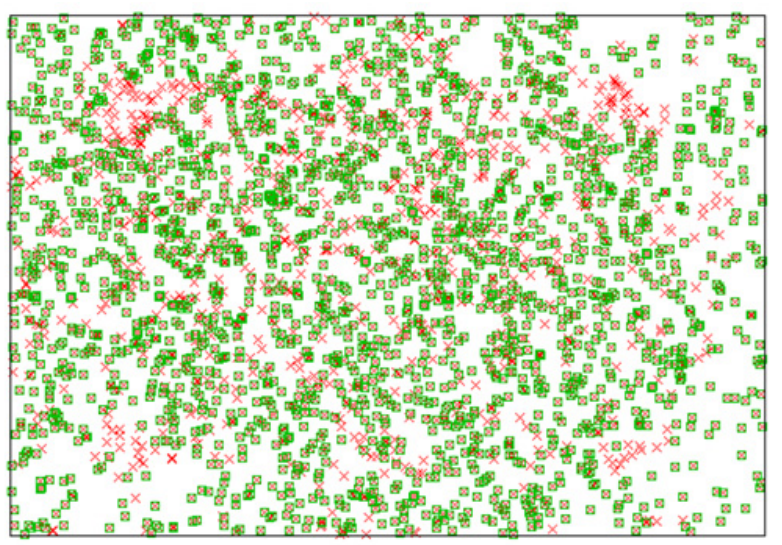

Figure 2. A plot of detected light sources (red crosses) and their identified catalog counterparts (green boxes) in the field of view of the wide-field camera of the Auger FRAM.

The FRAM will be integrated within the CTA software control system via an OPC standard interface to receive information about CTA field(s) of view and provide status information. On a lower level it will be operated using the RTS2 software [6] developed chiefly by Petr Kubánek. This complex open-source project for robotic observatories runs on any modern Linux system and is currently used at dozens of astronomical telescopes of various sizes (even at the 1-meter scale) around the world and allows autonomous observation with a plethora of supported hardware and different modes of target selection.

\section{FRAM data and their use within CTA}

In technical details, the analysis of images taken by the CTA FRAM will be very similar to that described in [4] for the Shoot-the-Shower program with the wide-field camera of the Auger FRAM. Here we outline just the basic principles.

We foresee that one image will be taken per minute with a 30 second exposure (the other 30 seconds are needed to read the image out from the camera and can be used to re-position the mount in case of splitarray observation). Each image will be calibrated and run through a star detection algorithm. Each detected lightsource will be then compared a catalog; the difference between the observed light flux and the catalog value is the basis for the determination of the current atmospheric extinction in the direction of each given star.

Nevertheless, there are many effects to consider before such a comparison can be drawn, most importantly the uneven field of view of the lens-camera system, color indexes of stars, catalog inaccuracies and the long-term development of the system sensitivity (dusting of the lens etc.). The long-term effect is particularly challenging to account for correctly, but affects only the absolute scale of the measurement; any spatial and temporal variation in the conditions (signifying the passage of clouds) is readily detected from relative changes in extinction.

The absolute response of the system can be continuously tracked during nights with stable conditions by observing the same stellar field as it passes through
Filter B
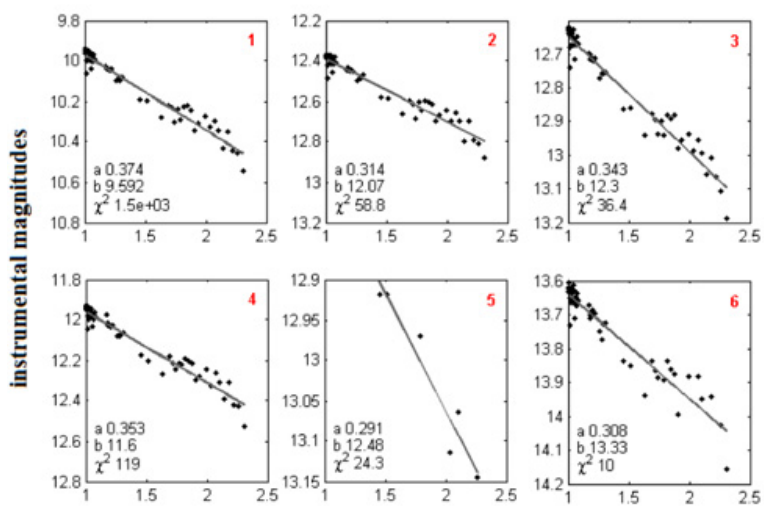

airmass

Figure 3. For six stars, measurements were made duting a snigle clear night at different zenith angles (and thus different amounts of airmass between the star and the telescope) and a linear function was fitted to the results. By extrapolating the function to zero, one can obtain the instrumental brightness of the stars unaffected by atmospheric conditions and thus, by comparison with the calatog, calibrate the absolute response of the telescope to starlight.

different zenith angles and fitting the zenith-angle dependence of extinction with a cosecans function (or, more accurately for high zenith angles, a linear function in airmass); additionally, very clear night identified by other instruments can be used as reference points.

The main deliverable product of FRAM for the CTA will be the maps of extinction across the current CTA field of view. These will be produced in real time (within a minute or two of the observation) for the purpose of scheduling and operative decisions. A more refined version (under routine review of the stability of the results and methods by an astronomer) will be available later to allow careful selection and analysis of the CTA data.

\section{Conclusions and outlook}

The "FRAM for CTA" project, based on a large body of experience obtained at the Pierre Auger Observatory, is in an advanced stage of development. Using similar hardware, software and analysis methods as tested on a device that has been continually running for almost 8 years ensures smooth implementation and reliable operation, much required within the highly ambitious CTA requirements. The use of stars as reliable, always available and non-disturbing calibration probes has a great potential for atmospheric monitoring (not only) at astroparticle physics projects.

We are grateful for the support by the grants of the Ministry of Education of the Czech Republic MSMT-CR LE13012 and LG14019. 


\section{References}

[1] Acharya, B.S. et al. (CTA Consortium), Astroparticle Physics 43, 3-18 (2013)

[2] Prouza, M. et al., Advances in Astronomy 2010, 849382 (2010)

[3] Abraham, J. et al. (The Pierre Auger Collaboration), Nucl. Instrum. Methods Phys. Res., Sect. A 523, 50 (2006)
[4] Ebr, J. et al. (The Pierre Auger Collaboration), Proceedings of the 3rd Astrorob Workshop, Rev. Mex. AA (SC) 45, 53 (2014)

[5] Ebr, J. et al., Proceedings of the 3rd Astrorob Workshop, Rev. Mex. AA (SC) 45, 114 (2014)

[6] Kubánek, P., Jelínek, M., Nekola, M. et al., GammaRay Bursts: 30 Years of Discovery, AIP Conference Proceedings 727, 753-756 (2004) 